\title{
Estudio Radiográfico Prospectivo de la Reparación Ósea en Sínfisis Mandibular Posterior a la Remoción Ósea de Mentón
}

\author{
Radiographic Prospective Study of Osseous Repair \\ in Mandibular Symphysis After Chin Bone Remove
}

"Claudio Ferreira Nóia; *Rafael Ortega-Lopes; "Fábio Ricardo Loureiro Sato; **Sergio Olate \& *Renato Mazzonetto

NÓIA, C. F.; ORTEGA-LOPES, R.; SATO, F. R. L.; OLATE, S. \& MAZZONETTO, R. Estudio radiográfico prospectivo de la reparación ósea en sínfisis mandibular posterior a la remoción ósea de mentón. Int. J. Morphol., 30(1):100-104, 2012.

RESUMEN: El retiro de hueso de sínfisis mandibular ha sido utilizado ampliamente para diferentes procedimientos reconstructivos maxilofaciales. El objetivo de esta investigación fue establecer la existencia de la reparación ósea en el defecto creado en sínfisis debido al retiro de hueso. Treinta pacientes ( 22 mujeres, 8 hombres) de entre 21 y 65 años fueron operados para retirar hueso de mentón que fue posteriormente aplicado en reconstrucción ósea alveolar; las cirugías fueron realizadas por dos cirujanos maxilofaciales y los pacientes fueron evaluados con telerradiografías en la etapa preoperatoria, postoperatoria inmediata (PIn) y postoperatoria tardía (PTar), donde se realizaron medidas horizontales y verticales del defecto óseo; los valores fueron estudiados con la prueba t de Student con valor de

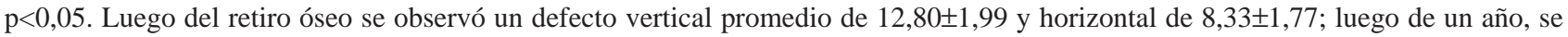
obtuvo una disminución de 32,8\% en el sentido vertical y 50,3\% horizontal, presentando significancia estadística en relación al PIn. Se concluye que existe reparación ósea del defecto originado en sínfisis siendo próximo al 30\%-50\% en la evaluación de un año posterior a la cirugía.

PALABRAS CLAVE: Sitios donante intraoral; Injerto óseo; Reparación ósea.

\section{INTRODUCCIÓN}

La reconstrucción ósea previo a la instalación de implantes óseointegrados es una técnica debidamente documentada con altas tazas de éxito (Deatherage, 2010). Del punto de vista biológico, la mejor opción reconstructiva está en el hueso autógeno (Deatherage; Olate et al., 2007a), existiendo algunas opciones intraorales que pueden ser utilizadas para retirar el hueso necesario; de entre ellos, la rama de mandíbula (Verdugo et al., 2009) y la sínfisis mandibular (Montazem et al., 2000) han sido popularizados por su capacidad de entregar amplias cantidades que permiten injertar en sitios que requieren instalación de implantes o reparar fisuras alveolares.

La sínfisis y parasínfisis mandibular ha sido utilizada por su capacidad de aportar hueso cortical y esponjoso y por el rápido acceso quirúrgico que presenta (Hoppenreijs et al., 1992), siendo aplicada en diferentes condiciones clínicas (Sindet-Pedersen \& Enemark, 1988; Precious \& Smith, 1992). Anatómicamente, esta región presenta estructuras importantes a considerar como los forámenes mentales con su paquete vascular y nervioso mental, músculos mentales y raíces dentarias (Montazem et al.), lo que justifica buena parte de las complicaciones y secuelas de esta cirugía. De esta forma, se ha identificado complicaciones postoperatorias como la parestesia regional, lesiones a rices dentarias, ptosis de labio, alteraciones en el contorno facial, entre otras (Raghoebar et al., 2001; Sbordone et al., 2009), que exigen del cirujano un amplio conocimiento de la técnica y de las condicionantes anatómicas del sector.

A pesar de todo, uno de los elementos poco estudiados hasta ahora es la reparación ósea que existe en el defecto creado en la sínfisis. Cuando se retira el hueso necesario, permanece un defecto en sínfisis que posteriormente debe repararse; de esta forma, el objetivo de esta investigación es identificar la reparación ósea existente en sínfisis mandibular luego de retirar un bloque óseo para reconstrucción alveolar.

\footnotetext{
* Area de Cirugía Oral y Maxilofacial, Departamento de Diagnóstico Oral, Facultad de Odontología de Piracicaba, Universidad Estadual de Campinas, Brasil. **Unidad de Cirugía Oral y Maxilofacial, Departamento de Odontología Integral, Facultad de Medicina, Universidad de La Frontera, Chile.
} 


\section{MATERIAL Y MÉTODO}

Fueron estudiados 30 pacientes (22 mujeres y 8 hombres) sometidos a remoción de hueso de mentón para cirugía de aumento de hueso alveolar con objetivo de instalar implantes óseointegrados; la edad media de los pacientes fue de 45 años (rango entre 21 y 65 años). Criterios de inclusión fueron la ausencia de patologías del metabolismo óseo u otra patología que altera la reparación ósea, quedando excluidos los pacientes con hábito tabáquico, alcohólicos o con historia de trauma. Esta investigación fue aprobada por el comité de ética de la Facultad de Odontología de la Universidad Estadual de Campinas con el numero 040/ 2009.

Procedimiento quirúrgico. Las cirugías fueron realizadas por dos cirujanos previamente calibrados. El acceso quirúrgico consistió de una incisión (hoja 15 en bisturí frio) realizada $5 \mathrm{~mm}$ inferior a la línea mucogingival iniciando en el sector derecho, inferior al canino ipsilateral y finalizó a nivel del canino del lado izquierdo; posteriormente fue realizada la incisión de los músculos mentales en la misma dirección hasta llegar a periostio, momento en el que fue rebatido un colgajo de espesor total con descolamiento total hasta la región del margen basilar de sínfisis. A continuación fueron observados los forámenes mentales del lado izquierdo y del lado derecho y fue establecido el límites de la osteotomía $5 \mathrm{~mm}$ inferior al ápice de los dientes inferiores, 5 $\mathrm{mm}$ superior al margen basilar de mandíbula y $5 \mathrm{~mm}$ hacia medial de ambos forámenes.

La osteotomía fue realizada con fresa tronco cónica No 702 montada en pieza de mano con rotación máxima de $25.000 \mathrm{rpm}$, bajo constante irrigación de suero fisiológico al $0,9 \%$. La longitud y magnitud de la osteotomía fue establecida en base a la necesidad de la reconstrucción, pero en todas ellas la osteotomía con fresa llego al área de hueso esponjoso; la remoción final del injerto fue realizada con cinceles curvos y rectos de $8 \mathrm{~mm}$ de ancho.

La síntesis fue realizada con tres puntos de sutura de tipo cat-gut cromado 3-0 en aguja de $17 \mathrm{~mm}$ traumática de 3/4 (Point Suture, Fortaleza-CE); los puntos simples fueron en el musculo mental del lado derecho, del lado izquierdo y un punto mediano, cuidando de mantener la adecuada reposición muscular. La síntesis de mucosa fue realizada mediante sutura continua con el uso de la misma sutura cat-gut 3-0. Posterior a la sutura fue instalado en región de mentón una cinta adhesiva con el objetivo de limitar el hematoma y edema presente; la cinta fue retirada a las $72 \mathrm{~h}$ de realizado el procedimiento.

Método de evaluación de imagen. Fueron realizados estudios con telerradiografía en la etapa preoperatoria (Pop), postoperatoria inmediata (PIn) (20-30 días postquirúrgico) y postoperatoria tardía (PTar) (12 meses postquirúrgico). Las imágenes radiográficas fueron captadas por el mismo operador y en el mismo equipo radiográfico. La telerradiografía inicial fue la base de las comparaciones radiográficas posteriores con la que se identificó el contorno óseo y de tejido blando (Fig. 1a).

Las medidas radiográficas fueron realizadas por un solo operador quien estableció su calibración inicial realizando el mismo procedimiento en dos momentos diferentes con 20 días de diferencia entre cada una; las medidas realizadas en la radiografía PIn y PTar fueron:

Vertical: distancia existente entre el margen supero-anterior del defecto óseo y el margen ínfero-anterior del mismo (medición en línea recta) (Fig. 1b).

Horizontal: distancia existente entre el punto más posterior del defecto óseo y la intersección con la línea vertical (Fig. 1c).

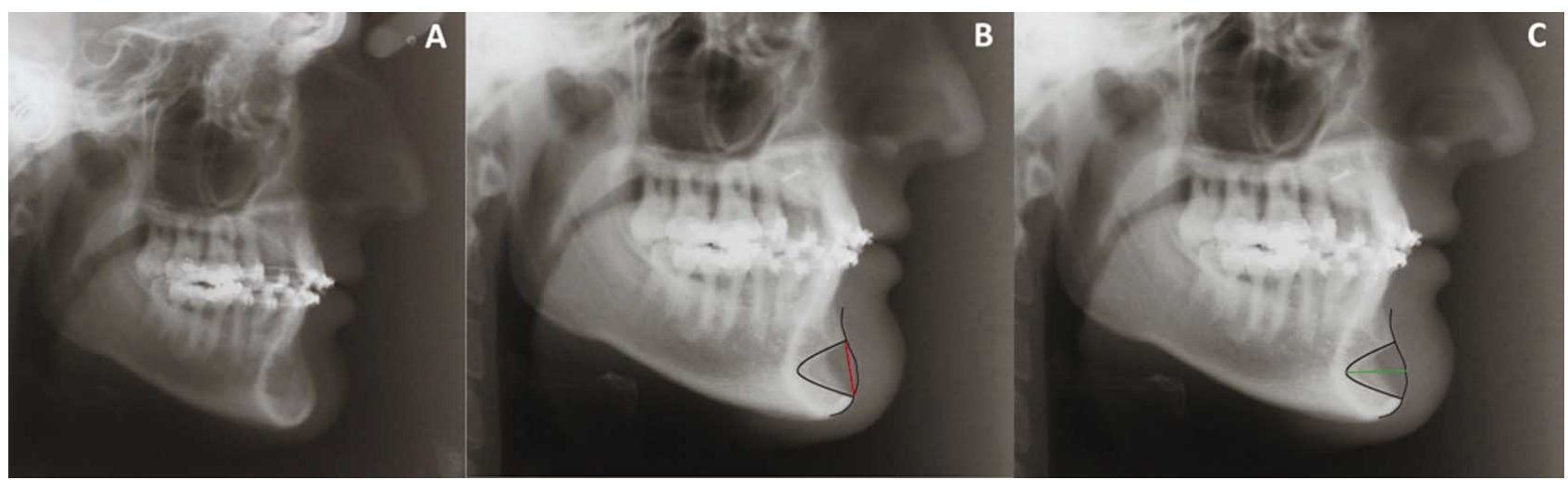

Fig. 1. (a) imagen radiográfica preoperatoria utilizada para realizar la evaluación inicial, (b) dirección de la medida vertical entre los puntos ya señalados en el PIn, (c) dirección de la medida horizontal entre la zona más posterior del defecto y el punto de contacto con la línea vertical en el PIn. 
Las mediaciones fueron realizadas sobre papel de acetato de $0,07 \mathrm{~mm}$ y lápiz de grafito de $0,5 \mathrm{~mm}$; cada medida fue realizada tres veces con una semana de diferencia entre cada medición y fue considerado como valor final el valor promedio de las mediciones realizadas.

Método estadístico. Inicialmente, los datos fueron manejados con el test de Léveme y el test de Kolmogorov-Smirnov donde se observó que la muestra era paramétrica; posteriormente se aplicó el test t de Student con un nivel de significancia de 5\% para establecer significancia estadística.

\section{RESULTADOS}

$\mathrm{Al}$ analizar el tamaño inicial del defecto óseo se observó en el periodo PIn presentaba una altura media de 12,8 $\pm 1,99 \mathrm{~mm}$ y una distancia anteroposterior de $8,33 \pm 1,77 \mathrm{~mm}$ (Tabla I). En

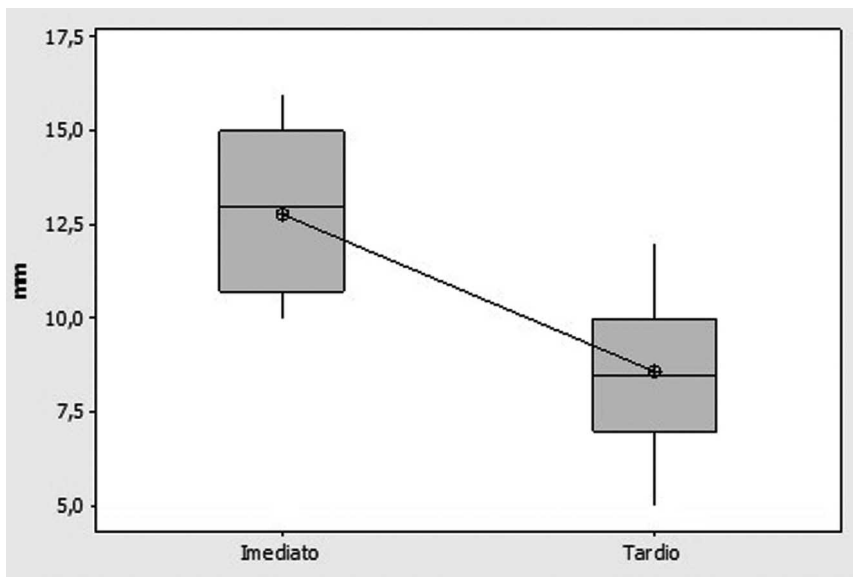

Fig. 2. comparación de los valores obtenidos en la medición vertical para el defecto en sínfisis en el PIn y el PTar.

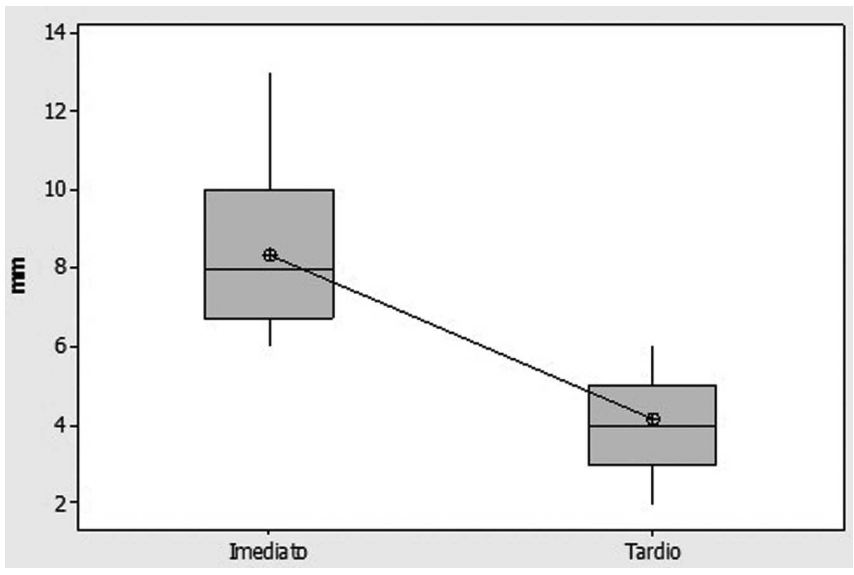

Fig. 3. comparación de los valores obtenidos en la medición horizontal de los defectos en sínfisis en el PIn y PTar. el periodo PTar presentaba una altura de $8,80 \pm 1.92 \mathrm{~mm}$ presentando una diferencia de aproximadamente $4 \mathrm{~mm}$, siendo estadísticamente significativo $(\mathrm{p}<0,05)$ con el PIn (Tabla II, Fig. 2). El defecto medido en el sentido horizontal en el PIn fue de 8,33 $\pm 1,77 \mathrm{~mm}$ mientras que en el PTar presentaba 4,17 $\pm 1,21 \mathrm{~mm}$, demostrando una disminución de aproximadamente $4 \mathrm{~mm}$, presentando una diferencia significativa $(p<0,05)$ en relación al PIn (Tabla II, Fig. 3).

Tabla I. Tamaño del defecto óseo creado a partir de la remoción de hueso desde la sínfisis mandibular en los 30 sujetos de muestra (según evaluación en la etapa postoperatoria inmediata).

\begin{tabular}{|c|c|c|c|c|}
\hline Paciente & Sexo & Edad & $\begin{array}{c}\text { Defecto } \\
\text { Vertical } \\
(\mathbf{m m})\end{array}$ & $\begin{array}{c}\text { Defecto } \\
\text { Horizontal } \\
(\mathbf{m m})\end{array}$ \\
\hline 1 & $\mathrm{~F}$ & 40 & 10 & 6 \\
\hline 2 & $\mathrm{~F}$ & 45 & 13 & 8 \\
\hline 3 & M & 21 & 15 & 10 \\
\hline 4 & $\mathrm{~F}$ & 34 & 12 & 9 \\
\hline 5 & $\mathrm{~F}$ & 42 & 14 & 7 \\
\hline 6 & $\mathrm{~F}$ & 55 & 13 & 8 \\
\hline 7 & M & 51 & 15 & 10 \\
\hline 8 & M & 60 & 10 & 10 \\
\hline 9 & $\mathrm{~F}$ & 48 & 14 & 13 \\
\hline 10 & F & 41 & 16 & 8 \\
\hline 11 & $\mathrm{~F}$ & 65 & 10 & 8 \\
\hline 12 & M & 54 & 13 & 9 \\
\hline 13 & $\mathrm{~F}$ & 45 & 10 & 6 \\
\hline 14 & $\mathrm{~F}$ & 48 & 15 & 8 \\
\hline 15 & $\mathrm{~F}$ & 56 & 13 & 10 \\
\hline 16 & M & 35 & 15 & 9 \\
\hline 17 & M & 39 & 14 & 10 \\
\hline 18 & $\mathrm{~F}$ & 30 & 13 & 9 \\
\hline 19 & F & 42 & 10 & 7 \\
\hline 20 & $\mathrm{~F}$ & 49 & 10 & 6 \\
\hline 21 & F & 52 & 15 & 6 \\
\hline 22 & M & 50 & 12 & 6 \\
\hline 23 & M & 62 & 12 & 10 \\
\hline 24 & F & 43 & 16 & 9 \\
\hline 25 & $\mathrm{~F}$ & 57 & 13 & 11 \\
\hline 26 & F & 33 & 10 & 6 \\
\hline 27 & F & 47 & 12 & 8 \\
\hline 28 & F & 28 & 13 & 8 \\
\hline 29 & $\mathrm{~F}$ & 40 & 15 & 9 \\
\hline 30 & $\mathrm{~F}$ & 38 & 11 & 6 \\
\hline Promedio & & 45 & 12,80 & 8,33 \\
\hline $\mathrm{DE}$ & & 10,5 & 1,99 & 1,77 \\
\hline
\end{tabular}


Tabla II. Distribución de la disminución del defecto óseo creado en la muestra de 30 sujetos. Difiere del PIn según el test t de Student $(\mathrm{p}<0,05)$.

\begin{tabular}{lccc}
\hline Defecto & $\begin{array}{c}\text { P. Imediato (1er mes) } \\
(\boldsymbol{X} \pm \mathbf{D E})\end{array}$ & $\begin{array}{c}\text { P. Tardio }\left(\mathbf{1 2}^{\circ} \text { mes }\right) \\
(\boldsymbol{X} \pm \mathbf{D E})\end{array}$ & \% Aprox. de Reparación \\
\hline Vertical & $12,80 \pm 1,99$ & $8,60 \pm 1,92^{*}$ & $32,8 \%$ \\
Horizontal & $8,33 \pm 1,77$ & $4,14 \pm 1,21^{*}$ & $50,3 \%$ \\
\hline
\end{tabular}

\section{DISCUSIÓN}

La reconstrucción ósea alveolar es reconocida como una técnica segura, eficaz, previsible y funcional (Olate et al., 2008). Los sitios donantes intraorales han determinado la posibilidad de realizar los procedimientos con anestesia local, lo cual optimiza los tiempos y disminuye los costos económicos del tratamiento (Olate et al., 2007b).

La morbilidad del retiro óseo de mentón ha sido documentada en diferentes publicaciones destacando la parestesia postoperatoria y las alteraciones de tejido blando tanto de mentón como de labio (Raghoebar et al.; Sbordone et al.). Los autores de la presente investigación creen que una causa probable para ocasionar estas alteraciones puede ser el tamaño del defecto óseo creado al momento del retiro óseo; si bien la sola osteotomía del sector puede generar los fenómenos de parestesia y alteraciones dentarias (Hoppenreijs et al.), la profundidad del defecto puede generar un disturbio importante en la reposición muscular de los músculos mentales.

La cantidad de hueso retirado de sínfisis puede ser variada y alcanzar valores cercanos a 20,9 X 9,9 X 6,9 mm (en promedio) para retiro de bloques, lo que implica una importante cantidad de hueso disponible para reconstrucción (Montazem et al.); de esta forma, la reparación del defecto creado puede ser altamente compleja dado el tamaño de la cavidad que permanece en la sínfisis mandibular.

La reparación ósea en esta área ha sido poco estudiada; sin embargo, el trabajo publicado por Dirk et al. (2010) señalo que la velocidad de reparación ósea en el defecto generado por el retiro óseo de sínfisis fue asociada directamente con la edad del paciente, siendo esta investigación realizada en individuos adolescentes y jóvenes. También señalaron que, después de un año de seguimiento, la reparación del sector fue cercana al $86 \%$ del defecto original observándose también un aumento estadísticamente significativo del tejido blando de mentón. La presente investigación trato pacientes cuya edad media estaba en 45 años, donde se observó una disminución del defecto vertical de 32,8\% y del defecto horizontal de $50,3 \%$ en un año de seguimiento. Ihan Hren \& Milajavec (2008) señalaron similares conclusiones respecto de la influen- cia de la edad del paciente y la reparación ósea, siendo menor en sujetos de mayor edad.

Por otra parte, la calidad del hueso reparado también puede ser objetivo de investigación; si bien en este trabajo no se estudiaron tales parámetros, los resultados de Ihan Hren \& Milajavec sugieren que el tejido óseo se presenta con un aspecto imagenológico normal al establecer un $88 \%$ de densidad ósea en defectos de entre $20 \mathrm{~mm}$ y $50 \mathrm{~mm}$ y un $97 \%$ de densidad ósea en defectos de entre $20 \mathrm{~mm}$ y $30 \mathrm{~mm}$ después de un año de seguimiento.

La presente investigación concluye que la reparación ósea posterior al retiro de hueso de sínfisis mandibular existe y es significativamente rápida al realizar el control a un año de la cirugía. A partir de estos resultados surgen dudas respecto de cómo se origina la reparación ósea, cuál es su influencia en las patologías neurosensoriales postoperatorias y cómo se comportan los tejidos blandos (especialmente músculos mentales) durante la fase de reparación ósea.

NÓIA, C. F.; ORTEGA-LOPES, R.; SATO, F. R. L.; OLATE, S. \& MAZZONETTO, R. Radiographic prospective study of osseous repair in mandibular symphysis after chin bone remove. Int. J. Morphol., 30(1):100-104, 2012.

SUMMARY: Bone harvest of mandibular symphysis has been used in maxillofacial reconstruction procedures. The aim of this research was to establish the presence of bone repair into osseous defect caused by removal of bone. Thirty patient s, 2 female and 8 male, ranging between 21 and 65 years of age underwent surgery for chin bone harvest and alveolar reconstruction. The surgery was performed by two maxillofacial surgeons and the patients were evaluated with lateral radiography in the pre-operatory stage, in early post-operatory and in late post-operatory stages. Horizontal and vertical measures of bone defect were realized; the dates were analyzed by Student $t$ test with a value of $\mathrm{p}<0.05$. After bone harvest, a vertical defect of $12.80 \pm 1.99$ was observed and a horizontal defect of $8.33 \pm 1.77$ was observed. After one year the defect decreased to $32.8 \%$ in vertical evaluation, and $50.3 \%$ in the horizontal evaluation with a statistical significance $(\mathrm{p}<0.05)$ in relation to the early postoperatory stage. Therefore, we conclude that in bone repair of mandibular symphysis defect is close to $30-50 \%$ in a one-year follow-up.

KEY WORDS: Intraoral donor; Bone graft; Bone repair. 


\section{REFERENCIAS BIBLIOGRÁFICAS}

Deatherage, J. Bone materials available for alveolar grafting. Oral Maxillofac. Surg. Clin. North. Am., 22:347-52, 2010.

Dirk, E. A.; de Ruiter, A. P.; Van der Bilt, A. \& Koole, R. Effect on the contour of bone and soft tissue one year after harvesting chin bone for alveolar cleft repair. Int. J. Oral Maxillofac. Surg., 39:962-7, 2010.

Hoppenreijs, T. J.; Nidjam, E. S. \& Freihofer, H. P. The chin as donor site in early secondary ostetoplasty: a retrospective clinical and radiological evaluation. J. Craniomaxillofac. Surg., 20:119-24, 1992.

Ihan Hren, N. \& Milijavec, M. Spontaneous bone healing of the large bone defects in the mandible. Int. J. Oral Maxillofac. Surg., 37:1111-6, 2008.

Montazem, A.; Valauri, D. V.; St-Hilaire, H. \& Buchhinder, D. The mandibular symphysis as a donor site in maxillofacial bone grafting: a quantitative anatomic study. J. Oral Maxillofac. Surg., 58:1368-71, 2000.

Olate, S.; Oliveira, G. R.; Jaimes, M. \& Albergaria Barbosa, J. $\mathrm{R}$. Recuperación ósea en procedimentos de reconstrucción y colocación de implantes. Int. J. Morphol., 25:649-57, 2007a.

Olate, S.; Kluppel, L. E.; Chaves Netto, H. D. M.; Stabile, G. A.; Mazzonetto, R. \& Albergaria-Barbosa, J. R. Sitios donantes mandibulares en implantologia - una evaluación clínica. Int. J. Odontostomat., 1:121-7, 2007b.

Olate, S.; Kluppel, L. E.; Chaves Netto, H. D. M.; Jaimes, M.; Albergaria-Barbosa, J. R. \& Mazzonetto, R. Reposición maxilar y reconstrucción con cresta iliaca. Parte 1 . Indicaciones y técnica quirúrgica. Int. J. Odontoestomat., 2:43-52, 2008.

Precious, D. S. \& Smith, W. P. The use of mandibular symphyseal bone in maxillofacial surgery. Br. J. Oral Maxillofac. Surg., 30:148-52, 1992.

Raghoebar, G.; Louwerse, C.; Kalk, W. \& Vissink, A. Morbidity of chin bone harvesting. Clin. Oral Impl. Res., 12:503-7, 2001.

Sbordone, L.; Menchini-Fabris, G. B.; Toti, P.; Sbordone, L.; Califano, L. \& Guidetti, F. Clinical survey of neurosensory side-effects of mandibular parasymphyseal bone harvesting. Int. J. Oral Maxillofac. Surg., 38:139-45, 2009.

Sindet-Pedersen, S. \& Enemark, H. Mandibular bone grafts for reconstruction of alveolar clefts. J. Oral Maxillofac. Surg., 46:533-7, 1988.
Verdugo, F.; Simonian, K.; Smith McDonald, R. \& Nowzari, H. Quantitation of mandibular ramus volume as a source of bone grafting. Clin. Implant Dent. Relat. Res., 11(Supp. 1):e32-7, 2009.

Dirección para correspondencia:

Claudio Ferreira Nóia

Área de Cirurgia Buco-Maxilo-Faciais FOP-Unicamp

Av. Limeira, 901, Areião

Piracicaba-São Paulo-Brasil CEP:13414-903

Tel: 551921065325

Email: claudionoia@fop.unicamp.br

Recibido : 02-07-2011

Aceptado: 21-11-2011 